\title{
CANONICAL CORRELATIONS FOR TARGET DETECTION IN A PASSIVE RADAR NETWORK
}

\author{
Yuan Wang ${ }^{1}$, Louis L. Scharf ${ }^{2}$, Ignacio Santamaría ${ }^{3}$, and Haonan Wang ${ }^{4}$ \\ ${ }^{1}$ Department of Mathematics and Statistics, Washington State University, Pullman, WA, USA \\ ${ }^{2}$ Department of Mathematics, ${ }^{4}$ Department of Statistics, Colorado State University, Fort Collins, CO, USA \\ ${ }^{3}$ Department of Communications Engineering, University of Cantabria, Santander, Spain \\ ywang@math.wsu.edu, scharf@engr.colostate.edu,nacho@gtas.dicom.unican.es, wanghn@stat.colostate.edu
}

\begin{abstract}
In this work, we consider a two-channel multiple-input multiple-output (MIMO) passive detection problem, in which there is a surveillance array and a reference array. The reference array is known to carry a linear combination of broadband noise and a subspace signal of known dimension but unknown basis. The question is whether the surveillance channel carries a linear combination of broadband noise and a subspace signal of unknown basis, which is correlated with the subspace signal in the reference channel. We consider a second-order detection problem where these subspace signals are structured by an unknown, but common, $p$-dimensional random vector of symbols transmitted from sources of opportunity, and then received through unknown $M \times p$ matrices at each of the $M$-element arrays. The noises in each channel have arbitrary spatial correlation. We derive the generalized likelihood ratio test (GLRT) statistic and show it is a monotone function of canonical correlations between the reference and surveillance channels.

Index Terms-Passive detection, MIMO channels, passive radar, generalized likelihood ratio, canonical coordinates.
\end{abstract}

\section{INTRODUCTION}

This paper is motivated by a passive radar application, where the problem is to determine if there are complex demodulations and synchronizations in several surveillance antennas (or antenna arrays) that bring signals in the surveillance antennas into coherence with signals in the reference antennas. This coherence is manifested in the synchronous sharing of transmit symbols from several opportunistic transmitters (e.g. digital television, digital audio broadcast, or mobile communication systems), and as a consequence there is correlation between signals observed at the MIMO surveillance array and the MIMO reference array. So the problem is to detect correlated subspace signals of dimension-p in two MIMO channels. In passive radar the signal paths for the reference and the surveillance channels are typically separated by digital beamforming using directional antennas.

The conventional approach for passive detection uses the cross-correlation (CC) between the data received in the reference and surveillance channels as the test statistic [1]. However, the noise in the reference signal renders the CC detection scheme suboptimal, especially in MIMO scenarios for which the inherent low-dimensional subspace structure of the transmitted signal can be exploited [2]. Passive MIMO target detection with a noisy reference channel has recently been considered in [3], where the transmitted waveform is considered as a deterministic unknown. The authors of [3] derived the generalized likelihood ratio test (GLRT) for this deterministic target model under spatially white noise of known variance. The work in [4] derives the GLRT in passive radar problem that models the received signal as a deterministic waveform scaled by an unknown single-input single-output (SISO) channel and under white noise of either known or unknown variance. A passive detector that exploits the low-rank structure of the received signal has been proposed in [5]. Instead of computing the cross-correlation between the surveillance and reference channel measurements, the ad-hoc detector proposed in [5] cross-correlates the dominant left singular vectors of the matrices containing the observations acquired at both channels.

Detection of a dimension-one subspace signal with a single array of sensors under white noise of unknown 
level has been addressed in [6], and extensions to diagonal noise covariance matrices and dimension- $p$ subspace signals can be found in [7]. Other variants of this problem, motivated by cognitive radio and multi-static radar applications, and notably based on averaging with respect to Haar measure on the space of dimension- $p$ subspaces, have been considered in [8] and the references therein. These detection problems are solved with a single array of sensors at the surveillance channel (for radar applications) or at the secondary user (for cognitive radio applications). The model considered in this paper is solved with the assistance of an additional multi-antenna reference channel which acquires a noisy and distorted version of the transmitted signal.

In this paper, we address the MIMO passive detection problem in a multivariate normal model when the surveillance and reference channels are equipped with $M$ antennas, the transmitted signal is an unknown rank- $p$ signal, and the noises at surveillance and reference channels are uncorrelated between them, but each having different spatial correlation models. It turns out that this is a problem in factor analysis [9], where there are constraints on the factor loadings and the factors. The problem may be viewed as a onechannel factor analysis problem with constraints on the factor loadings under the null hypothesis, or as a twochannel factor analysis problem, with constraints on the factor loadings under the null, and with common factors under the alternative. We derive the generalized likelihood ratio test (GLRT) as a ratio of determinants of covariance matrices. In the general case when the noise covariaces are unstructured, the test statistic is a monotone function of canonical correlations between the reference and surveillance channels.

Notation: The superscripts $(\cdot)^{T}$ and $(\cdot)^{H}$ denote transpose and Hermitian, respectively. The determinant, trace and Frobenius norm of a matrix $\mathbf{A}$ will be denoted, respectively, as $\operatorname{det}(\mathbf{A}), \operatorname{tr}(\mathbf{A})$ and $\|\mathbf{A}\|_{F} \cdot \mathbf{I}_{M}$ is the identity matrix of dimensions $M \times M$, and $\mathbf{0}$ denotes either a column vector with $M$ zeros, or the zero matrix of appropriate dimensions (the difference should be clear from the context). We use $\mathbf{A}^{1 / 2}\left(\mathbf{A}^{-1 / 2}\right)$ to denote the square root matrix of the Hermitian matrix $\mathbf{A}\left(\mathbf{A}^{-1}\right) ; \operatorname{diag}_{M}(\mathbf{A})$ is a block-diagonal matrix formed by $M \times M$ blocks on the diagonal of $\mathbf{A}$. The expectation operator will be denoted by $E[\cdot]$, and $\mathbf{x} \sim \mathcal{C N}_{M}(\mathbf{0}, \mathbf{R})$ indicates that $\mathbf{x}$ is an $M$-dimensional complex circular Gaussian random vector of zero mean and covariance $\mathbf{R}$.

\section{PROBLEM FORMULATION}

Our two-channel measurement model is

$$
\left[\begin{array}{c}
\mathbf{x}_{s}[n] \\
\mathbf{x}_{r}[n]
\end{array}\right]=\left[\begin{array}{c}
\theta \mathbf{H}_{s} \\
\mathbf{H}_{r}
\end{array}\right] \mathbf{s}[n]+\left[\begin{array}{c}
\mathbf{v}_{s}[n] \\
\mathbf{v}_{r}[n]
\end{array}\right] ; \quad n=1,2, \ldots, N
$$

where $\mathbf{x}_{s}[n] \in \mathcal{C}^{M}$ and $\mathbf{x}_{r}[n] \in \mathcal{C}^{M}$ are the surveillance and reference measurements; $\mathbf{s}[n] \in \mathcal{C}^{p}$ contains the signal transmitted by $p$ opportunistic illuminators, $\mathbf{H}_{s} \in \mathbb{C}^{M \times p}$ and $\mathbf{H}_{r} \in \mathbb{C}^{M \times p}$ represent the $M \times p$ channels from the transmitter(s) to the surveillance and reference multiantenna receivers, respectively. The parameter $\theta \in\{0,1\}$ determines whether or not there is a signal $\mathbf{H}_{s} \mathbf{s}[n]$ in the surveillance channel.

We treat the symbol sequence as a sequence of circular, Gaussian random vectors with unknown covariance $E\left[\mathbf{s}[n] \mathbf{s}[m]^{H}\right]=\mathbf{C} \delta[n-m]$. The factor loadings $\mathbf{H}_{s}$ and $\mathbf{H}_{r}$ are unknown, to be identified in a maximum likelihood procedure. Without loss of generality, the symbol covariance may be absorbed into these factor loadings and thus we assume $\mathbf{C}=\mathbf{I}_{p}$. The vectors $\mathbf{v}_{s}[n]$ and $\mathbf{v}_{r}[n]$ model the additive noise. For notational convenience, the signal, noise, and channel vectors can be stacked as $\mathbf{x}[n]=\left[\mathbf{x}_{s}[n]^{T}, \mathbf{x}_{r}[n]^{T}\right]^{T}$, $\mathbf{v}[n]=\left[\mathbf{v}_{s}[n]^{T}, \mathbf{v}_{r}[n]^{T}\right]^{T}$ and $\mathbf{H}=\left[\mathbf{H}_{s}^{T}, \mathbf{H}_{r}^{T}\right]^{T}$, respectively. The additive noise is assumed to be temporally white, zero-mean Gaussian distributed, and uncorrelated between the surveillance and reference channels. The noise covariance matrix can then be written as

$$
E\left[\mathbf{v}[n] \mathbf{v}[m]^{H}\right]=\left[\begin{array}{cc}
\boldsymbol{\Sigma}_{s s} & \mathbf{0} \\
\mathbf{0} & \boldsymbol{\Sigma}_{r r}
\end{array}\right] \delta[m-n]
$$

where $\boldsymbol{\Sigma}_{s s}$ and $\boldsymbol{\Sigma}_{r r}$ are arbitrary psd matrices:

The passive detection problem is to test the hypothesis that the surveillance channel contains no signal, versus the alternative that it does:

$$
\begin{aligned}
& \mathcal{H}_{0}: \theta=0 \\
& \mathcal{H}_{1}: \theta=1
\end{aligned}
$$

Denote by $\mathcal{R}_{0}$ and $\mathcal{R}_{1}$ the set of measurement covariance matrices under the null hypothesis and alternative hypothesis, respectively. We have

$$
\begin{aligned}
& \mathcal{R}_{0}=\left\{\left[\begin{array}{ll}
\mathbf{0} & \mathbf{0} \\
\mathbf{0} & \mathbf{H}_{r} \mathbf{H}_{r}^{H}
\end{array}\right]+\left[\begin{array}{cc}
\boldsymbol{\Sigma}_{s s} & \mathbf{0} \\
\mathbf{0} & \boldsymbol{\Sigma}_{r r}
\end{array}\right]\right\} \\
& \mathcal{R}_{1}=\left\{\left[\begin{array}{ll}
\mathbf{H}_{s} \mathbf{H}_{s}^{H} & \mathbf{H}_{s} \mathbf{H}_{r}^{H} \\
\mathbf{H}_{r} \mathbf{H}_{s}^{H} & \mathbf{H}_{r} \mathbf{H}_{r}^{H}
\end{array}\right]+\left[\begin{array}{cc}
\boldsymbol{\Sigma}_{s s} & \mathbf{0} \\
\mathbf{0} & \boldsymbol{\Sigma}_{r r}
\end{array}\right]\right\} .
\end{aligned}
$$


This detection problem essentially amounts to testing between two different structures for the composite covariance matrix under the null hypothesis and alternative hypothesis. It can be written as

$$
\begin{aligned}
& \mathcal{H}_{0}: \mathbf{x}[n] \sim \mathcal{C N}_{2 M}(\mathbf{0}, \mathbf{R}), \mathbf{R} \in \mathcal{R}_{0} \\
& \mathcal{H}_{1}: \mathbf{x}[n] \sim \mathcal{C N}_{2 M}(\mathbf{0}, \mathbf{R}), \quad \mathbf{R} \in \mathcal{R}_{1} .
\end{aligned}
$$

There are two possible interpretations of this model: (1) it is a one-channel factor model with special constraint on the loadings under $\mathcal{H}_{0}$; or (2) it is a two channel factor model with constraint under $\mathcal{H}_{0}$ and common factors in the two channels.

\section{THE GENERALIZED LIKELIHOOD RATIO}

Let us now consider $N$ consecutive array snapshots under a model with generic covariance matrix $\mathbf{R}$

$$
[\mathbf{x}[1] \quad \ldots \quad \mathbf{x}[N]]=\mathbf{X} \in \mathcal{C}^{2 M \times N},
$$

which are i.i.d. realizations of $\mathbf{x}[n] \sim \mathcal{C N}_{2 M}(\mathbf{0}, \mathbf{R})$. As there are unknown parameters under both hypotheses, the Neyman-Pearson detector is not implementable for this composite test. Therefore, we adopt a generalized likelihood ratio test (GLRT), which usually results in simple detectors with good performance [9]. The likelihood may be written as

$$
f(\mathbf{X} ; \mathbf{R})=\frac{1}{\pi^{2 M N} \operatorname{det}(\mathbf{R})^{N}} \exp \left\{-N \operatorname{tr}\left(\mathbf{S R}^{-1}\right)\right\}
$$

where $\mathbf{S}=\frac{1}{N} \mathbf{X} \mathbf{X}^{H}$ is the sample covariance matrix, partitioned as

$$
\mathbf{S}=\left[\begin{array}{ll}
\mathbf{S}_{s s} & \mathbf{S}_{s r} \\
\mathbf{S}_{s r}^{H} & \mathbf{S}_{r r}
\end{array}\right]
$$

Here $\mathbf{S}_{s s}$ is the sample covariance matrix of the surveillance channel and the other blocks are defined similarly. The generalized likelihood ratio (GLR) is

$$
\Lambda=\frac{f\left(\mathbf{X} ; \hat{\mathbf{R}}_{1}\right)}{f\left(\mathbf{X} ; \hat{\mathbf{R}}_{0}\right)},
$$

where $\hat{\mathbf{R}}_{0}$ and $\hat{\mathbf{R}}_{1}$ are, respectively, the maximum likelihood (ML) estimates of the covariance matrix for model $j$ under $\mathcal{H}_{0}$ and $\mathcal{H}_{1}$. They maximize the loglikelihood function

$$
\mathcal{L}(\mathbf{R})=\log \operatorname{det}\left(\mathbf{S R}^{-1}\right)-\operatorname{tr}\left(\mathbf{S R}^{-1}\right),
$$

The GLRT for noise model $j$ reduces to

$\log (\Lambda)=\log \left(\frac{\operatorname{det}\left(\hat{\mathbf{R}}_{0}\right)}{\operatorname{det}\left(\hat{\mathbf{R}}_{1}\right)}\right)-\operatorname{tr}\left(\mathbf{S}\left(\hat{\mathbf{R}}_{1}^{-1}-\hat{\mathbf{R}}_{0}^{-1}\right)\right) \underset{H_{0}}{\stackrel{H_{1}}{\gtrless}} \eta$,

with $\eta$ a suitable threshold.

The ML estimate of the covariance matrix under the null is given by

$$
\hat{\mathbf{R}}_{0}=\operatorname{diag}_{M}(\mathbf{S})=\left[\begin{array}{cc}
\mathbf{S}_{s s} & \mathbf{0} \\
\mathbf{0} & \mathbf{S}_{r r}
\end{array}\right] .
$$

Under the alternative, the ML estimate has been derived for $p=1$ in [2].To present the result for general $p$, let $\mathbf{C}=\mathbf{S}_{s s}{ }^{-1 / 2} \mathbf{S}_{s r} \mathbf{S}_{r r}{ }^{-H / 2}$ be the sample coherence matrix between the surveillance and reference channels, and let $\mathbf{C}=\mathbf{F K G}^{H}$ be its singular value decomposition (SVD), where the matrix $\mathbf{K}=\operatorname{diag}\left(k_{1}, \cdots, k_{M}\right)$ contains the sample canonical correlations $1 \geq k_{1} \geq$ $\cdots \geq k_{M} \geq 0$ along its diagonal. The ML estimate is

$$
\hat{\mathbf{R}}_{1}=\left[\begin{array}{cc}
\mathbf{S}_{s s} & \mathbf{S}_{s s}^{1 / 2} \mathbf{C}_{p} \mathbf{S}_{r r}^{1 / 2} \\
\mathbf{S}_{r r}^{1 / 2} \mathbf{C}_{p}^{H} \mathbf{S}_{s s}^{1 / 2} & \mathbf{S}_{r r}
\end{array}\right]
$$

where $\mathbf{C}_{p}=\mathbf{F K}_{p} \mathbf{G}^{H}$ and $\mathbf{K}_{p}=$ $\operatorname{diag}\left(k_{1}, \cdots, k_{p}, 0, \ldots, 0\right)$ is a rank- $p$ truncation of $\mathbf{K}$. As a result, the GLRT is

$$
\Lambda=\operatorname{det}\left(\mathbf{I}-\mathbf{K}_{p}^{2}\right)^{-1}=\prod_{i=1}^{p} \frac{1}{\left(1-k_{i}^{2}\right)} \underset{H_{0}}{\stackrel{H_{1}}{\gtrless}} \eta,
$$

where $k_{i}$ is the $i$-th sample canonical correlation between the surveillance and reference channels, and $\eta$ is a suitable threshold. This formula was also derived in [10] for a different problem. Equation (14) has an interesting interpretation: $1-\Lambda_{4}{ }^{-1}$ is the coherence statistic, $0 \leq 1-\prod_{i=1}^{p}\left(1-k_{i}^{2}\right) \leq 1$.

Remark 1: Connection to Generalized Coherence. If the covariance matrix under $\mathcal{H}_{0}$ were assumed only block diagonal and under $\mathcal{H}_{1}$ it were assumed an arbitrary PSD matrix, the GLRT statistic would be the following generalized Hadamard ratio:

$$
H=\frac{\operatorname{det}(\mathbf{S})}{\operatorname{det}\left(\mathbf{S}_{s s}\right) \operatorname{det}\left(\mathbf{S}_{r r}\right)}=\prod_{i=1}^{M}\left(1-k_{i}^{2}\right)
$$

Notice also that $1-H$ is the Generalized Coherence (GC) originally defined in [11], and widely applied to multi-channel detection problems. So the net of prior knowledge of rank $p$ is to replace $M$ by $p$ in the coherence statistic. 
Remark 2: The detection problem and its GLRT are invariant to the transformation group $\mathcal{G}=\{G \mid$ $G(\mathbf{X})=\mathbf{T X Q}\}$, where the $2 M \times 2 M$ matrix $\mathbf{T}$ is a diagonal matrix of nonsingular $M \times M$ matrices $\mathbf{T}_{1}, \mathbf{T}_{2}$, and $\mathbf{Q}$ is an $N \times N$ unitary matrix. As a special case of this general invariance, the coherence detector is CFAR against unknown noise power in the surveillance chanel and unknown signal-plus-noise power in the reference channel. This is quite obviously a desirable property.

\section{SIMULATION STUDIES}

In this section we evaluate the performance of the GLR detectors by means of Monte Carlo simulations. The input signal-to-noise-ratio (SNR) for both the surveillance and reference channels is defined as

$$
\mathrm{SNR}_{i}=10 \log _{10} \frac{\operatorname{tr}\left(\mathbf{H}_{i}^{H} \mathbf{H}_{i}\right)}{\operatorname{tr}\left(\boldsymbol{\Sigma}_{i i}\right)}, \quad i=\{s, r\} .
$$

For given values of $\mathrm{SNR}_{s}$ and $\mathrm{SNR}_{r}$, the probability of detection, $P_{d}$, and probability of false alarm, $P_{f a}$, are estimated by averaging $10^{4}$ independent simulations, where at each simulation a different realization of the unknowns $\left(\mathbf{H}_{r}, \mathbf{H}_{\mathbf{s}}, \boldsymbol{\Sigma}_{s s}, \boldsymbol{\Sigma}_{r r}\right)$ is generated.

Fig. 1 depicts the ROC for the coherence detector for $p=1$ (dimension-one subspace signal), $M=5$ antennas and $N=100$ snapshots. The curves around the chance line are ROCs for detectors, not discussed here, which are mis-matched to the assumed noise model.

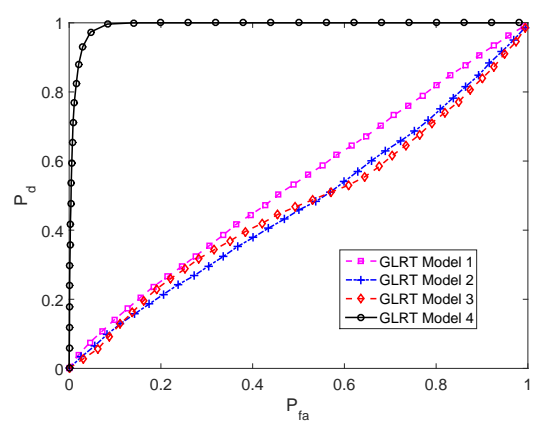

Fig. 1. ROC for coherence (or canonical correlation) detector, with $M=5$ antennas, $p=1, N=100$ snapshots and $\mathrm{SNR}_{s}=\mathrm{SNR}_{r}=-4 \mathrm{~dB}$.

\section{CONCLUSION}

In this paper we have addressed a problem motivated by passive radar. The problem is to detect a common subspace signal in two MIMO channels. It turns out that the problem is a problem in factor analysis, where there are constraints on the factor loadings and the factors. The problem may be viewed as a one-channel factor analysis problem with constraints on the factor loadings under the null hypothesis, or as a two-channel factor analysis problem, with constraints on the factor loadings under the null, and with common factors under the alternative. The GLRT compares the product $\prod_{1}^{p}\left(1-k_{i}^{2}\right)$ to a threshold, where the $k_{i}$ 's are squared canonical coordinates of the two channel sample covariance matrix. The product may be replaced by $1-\prod_{1}^{p}\left(1-k_{i}^{2}\right)$, which is coherence, so that the detector is a coherence detector.

\section{REFERENCES}

[1] J. Liu, H. Li, and B. Himed, "On the performance of the cross-correlation detector for passive radar applications," Signal Process., vol. 113, pp. 3237, 2015.

[2] I. Santamaria, L. L. Scharf, D. Cochran, and J. Via, "Passive detection of rank-one signals with a multiantenna reference signal," in European Signal Processing Conference (EUSIPCO), Budapest, Hungary, Sep. 2016.

[3] D. E. Hack, L. K. Patton, B. Himed, and M. A. Saville, "Detection in passive MIMO radar networks," IEEE Trans. Signal Process., vol. 62, no. 11, pp. 2999-3012, Jun. 2014.

[4] G. Cui, J. Liu, H. Li, and B. Himed, "Target detection for passive radar with noisy reference channel," in Proceedings of 2014 IEEE Radar Conference, Cincinnati (OH), USA, May 2014.

[5] S. Gogineni, P. Setlur, M. Rangaswamy, and R. R. Nadakuditi, "Random matrix theory inspired passive bistatic radar detection of low-rank signals," in IEEE Int. Conf. Acoust., Speech and Signal Proc. (ICASSP), Brisbane, Australia, Apr. 2015.

[6] O. Besson, S. Kraut, and L. Scharf, "Detection of an unknown rank-one component in white noise," IEEE Trans. Signal Process., vol. 54, no. 7, pp. 2835-2839, Jul. 2006.

[7] D. Ramirez, G. Vazquez-Vilar, R. LopezValcarce, J. Via, and I. Santamaria, "Detection of rank-P signals in cognitive radio networks 
with uncalibrated multiple antennas," IEEE Trans. Signal Process., vol. 59, no. 8, pp. 3764-3774, Aug. 2011.

[8] S. Sirianunpiboon, S. D. Howard, and D. Cochran, "Multiple-channel detection of signals having known rank," in IEEE Int. Conf. Acoust., Speech and Signal Proc. (ICASSP), Vancouver, Canada, May 2013, pp. 6536-6540.

[9] K. V. Mardia, J. T. Kent, and J. M. Bibby, Multivariate Analysis. Academic Press, 1979.

[10] P. Stoica, K. M. Wong, and Q. Wu, "On a nonparametric detection method for array signal processing in correlated noise fields," IEEE Trans. Signal Process., vol. 44, no. 4, pp. 1030-1032, Apr. 1996.

[11] D. Cochran, H. Gish, and D. Sinno, "A geometric approach to multiple channel signal detection," IEEE Trans. Signal Process., vol. 43, pp. 20492057, Sep. 1995. 